\title{
基于谱间相关性的高光谱图像稀疏分解算法
}

\author{
王 丽, 王 威, 刘勃妮 \\ (西安航空学院 电子工程学院, 陕西 西安 710077)
}

\begin{abstract}
摘要: 针对高光谱图像邻近波段相关性强的特点, 结合粒子群优化算法的快速寻优能力, 提出一种基 于谱间相关性的高光谱图像稀疏分解算法。将高光谱图像分组为参考波段图像和普通波段图像，参考 波段图像采用粒子群寻优找到最优原子, 实现稀疏分解。普通波段图像的最优原子由两部分构成, 一 部分原子从参考波段图像的最优原子继承而来，继承个数由普通波段图像与参考波段图像的谱间相关 性确定, 其余原子则由粒子群搜索得到。对高光谱数据集进行稀疏分解, 验证算法的分解效率, 结果 表明，在重构图像精度相当的条件下，稀疏分解速度比正交匹配追踪算法快约 18 倍。
\end{abstract}

关键词: 稀疏分解; 谱间相关性; 粒子群优化; 正交匹配追踪; 高光谱图像

中图分类号：TP301.6 文献标志码：A 文章编号：1001-8891(2020)10-0969-09

\section{Sparse Decomposition of Hyperspectral Images Based on Spectral Correlation}

\author{
WANG Li, WANG Wei, LIU Boni \\ (Department of Electronic Engineering, Xi'an Aeronautical University, Xi'an 710077, China)
}

\begin{abstract}
Considering the strong correlation between adjacent band images of hyperspectral data in combination with the fast searching ability of the particle swarm optimization algorithm, a sparse decomposition algorithm of hyperspectral images based on spectral correlation is proposed. The hyperspectral images are divided into reference and common band images. Particle swarm optimization is performed on the reference band images to find the optimal atoms and realize their sparse decomposition. The optimal atoms of a common band image consist of two parts. Parts of these atoms are inherited from the optimal atoms of the reference band images, and the number of inheritances is determined by the spectral correlation between the common and reference band images. The remaining atoms are obtained using particle swarm optimization. The experimental results on hyperspectral data show that in cases with the same reconstruction accuracy, the sparse decomposition rate is approximately 18 times higher than the orthogonal matching pursuit algorithm.
\end{abstract}

Key words: sparse decomposition, spectral correlation, particle swarm optimization, orthogonal matching pursuit, hyperspectral image

\section{0 引言}

高光谱图像包含丰富的二维空间几何信息和一维 光谱信息,适用于目标探测与识别、图像分类等领域 ${ }^{[1-5]}$, 信息量的剧增给数据的存储传输带来挑战。压缩感知 理论指出，在满足信号稀疏或可压缩的前提条件下， 信号的采样能够突破传统奈奎斯特定律的限制, 大大 降低数据量, 因此, 应用压缩感知理论处理高光谱图 像得到众多研究者的关注 ${ }^{[6-8]}$, 首要工作是实现稀疏表
示, 后续工作是实现稀疏表示下的高光谱图像去噪、 分类、解混 ${ }^{[9-16]}$ 等。常用的稀疏分解算法包括正交匹配 追踪 (orthogonal matching pursuit, OMP) ${ }^{[17-18]}$ 和基于 线性规划的基寻踪算法 (basis pursuit, BP) ${ }^{[19]}$, OMP 算法由于收玫快且精度高被大量采用。采用咒余字典 对高光谱图像信号进行稀疏表示时, 算法的复杂度较 高, 给稀疏分解算法的计算效率提出新的挑战。在考 虑进化算法寻优能力较强的思想下, 作者利用粒子群 优化 (particle swarm optimization, PSO) 对 OMP 算法 
进行改进, 提出了基于粒子群优化的稀疏分解算法 ( particle swarm optimization algorithm based on orthogonal matching pursuit, PSO_OMP) ${ }^{[20]}$ 。在保证 重构精度的条件下, 利用谱间相关性强去进一步提高 稀疏分解效率是本文的研究问题。

\section{1 基于谱间相关性的稀疏分解算法}

\section{1 基本框架}

高光谱图像的光谱分辨率高, 邻近波段具有极强 的相关性，但相邻波段之间的谱间相关性会随着波段 间距的增大而减小，只有一定间距内的波段会保持较 大的相关性。在稀疏分解过程中, 借鉴高光谱图像分 组处理的思想 ${ }^{[21]}$, 将所有高光谱图像分为参考波段图 像和非参考波段图像, 采用不同的最优原子搜索方 式，提高稀疏分解的计算效率。

高光谱图像数据表示为 $X \in R^{N \times N_{\lambda}}, N=N_{\mathrm{r}} \times N_{\mathrm{c}}$, $N_{\mathrm{r}}$ 和 $N_{\mathrm{c}}$ 分别表示单个波段图像的行数和列数, $N_{\lambda}$ 表 示波段数, $X_{b}$ 是第 $b$ 个波段图像的向量化表示, $b=$ $1,2, \cdots, N_{\lambda}$ 对 $N_{\lambda}$ 个波段进行均匀连续分组, 即每组 所包含的波段个数相同, 波段分组的示意图如图 1 所 示 ${ }^{[21]}$ 。其中, $G_{\mathrm{s}}$ 表示分组大小, $G_{\mathrm{n}}$ 表示组号, 整个 高光谱图像包含 $\left\lfloor N_{\lambda} / G_{\mathrm{s}}\right\rfloor$ 个完整的组, $\lfloor\cdot\rfloor$ 表示向下取 整, 最后一组包含 $N_{\lambda}-G_{\mathrm{s}}\left\lfloor N_{\lambda} / G_{\mathrm{s}}\right\rfloor$ 个波段图像。每组包 含一个参考波段图像和 $G_{\mathrm{s}}-1$ 个非参考波段图像, 组 内的波段图像序号用 $j$ 表示, $j=1$ 表示其为参考波段 图像, 用 $X_{G \mathrm{n}, \mathrm{ref}}$ 表示, $j=2, \cdots, G_{\mathrm{s}}$ 表示其为非参考波 段图像, 用 $X_{G \mathrm{n}, 2}, X_{G \mathrm{n}, 3}, \cdots, X_{G \mathrm{n}, j}, \cdots, X_{G \mathrm{n}, G \mathrm{~s}}$ 表示, 第 $G_{\mathrm{n}}$ 组的第 $j$ 个波段是原高光谱图像的第 $\left(\left(G_{\mathrm{n}}-1\right) G_{\mathrm{s}}+j\right)$ 个波段。 $G_{\mathrm{n}}$ 和 $G_{\mathrm{n}}+1$ 是两个相邻的组号, 其对应的参

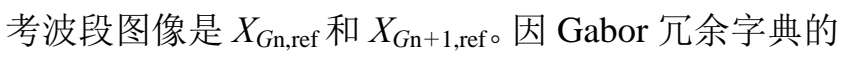
圥余度高, 若图像大小为 $256 \times 256$, 则 Gabor 字典的 原子个数将达到 57933772 , 计算时间将难以承受。在 考虑计算能力的条件下, 对图像进行分块处理, 分块 大小为 $B$, 参考波段图像块和非参考波段图像块的向 量化表示分别为 $X_{G \mathrm{n}, \mathrm{ref}, b l}$ 和 $X_{G \mathrm{n}, j, b l}, b l=1,2, \cdots, N / B^{2}$ 。
基于谱间相关性的稀疏分解算法基本框架如图 2 所示, 图中仅给出一个分组内单个参考波段图像块和 非参考波段图像块的稀疏分解过程。参考波段图像块 和非参考波段图像块的稀疏分解过程均采用 OMP 算 法的框架, 即每次迭代过程中, 通过一定的方式寻找 到最优原子并进行残差更新, $k$ 是 OMP 算法的迭代次 数。针对参考波段图像块, 采用 PSO 的方式快速搜索 到最优原子, 经过多次分解后得到最优原子集合, 并 利用搜索到的最优原子得到重构的参考波段图像块 为 $\hat{X}_{G \mathrm{n}, \mathrm{ref}, b l}$, 完成稀疏分解, 其稀疏分解的详细过程 参见文献[20], 此处不再赘述。

非参考波段图像块与参考波段图像块具有强相关 性, 二者具有共性特性, 可用相同原子进行稀疏表示。 在非参考波段图像块的最优原子搜索过程中，从参考 波段图像块的最优原子集合中继承一部分最优原子, 用这些最优原子对非参考波段图像块进行稀疏表示 后, 将此稀疏表表示后的残差作为需要稀疏分解的信 号, 再采用 PSO 搜索得到另一部分最优原子, 最后形 成最优原子集合并最终得到重构的非参考波段图像 块 $\hat{X}_{G \mathrm{n}, j, b l}$ 。所提算法在分析高光谱图像特性的基础 上, 借助粒子群优化和谱间相关性对 OMP 算法进行 改进, 标记为 MPSO_OMP (Modified PSO_OMP)。

\section{2 稀疏分解过程}

参考波段图像利用 PSO_OMP 算法完成稀疏分 解, 得到最优原子集合表示为:

$$
\Theta_{K}=\left[g_{\gamma_{0}}, g_{\gamma_{2}}, \cdots, g_{\gamma_{k}}, \cdots, g_{\gamma_{K-1}}\right]
$$

式中: $K$ 是最优原子的个数。

非参考波段图像与参考波段图像具有极强的相 关性, 表明两个图像之间具有共性特征, 可用相同的 原子进行稀疏表示。非参考波段图像的部分最优原子 可从参考波段图像的最优原子集合中继承而来，继承 个数 $K_{\text {in }}$ 表示为:

$$
K_{\text {in }}=\operatorname{round}(\sigma \times h \times K)
$$

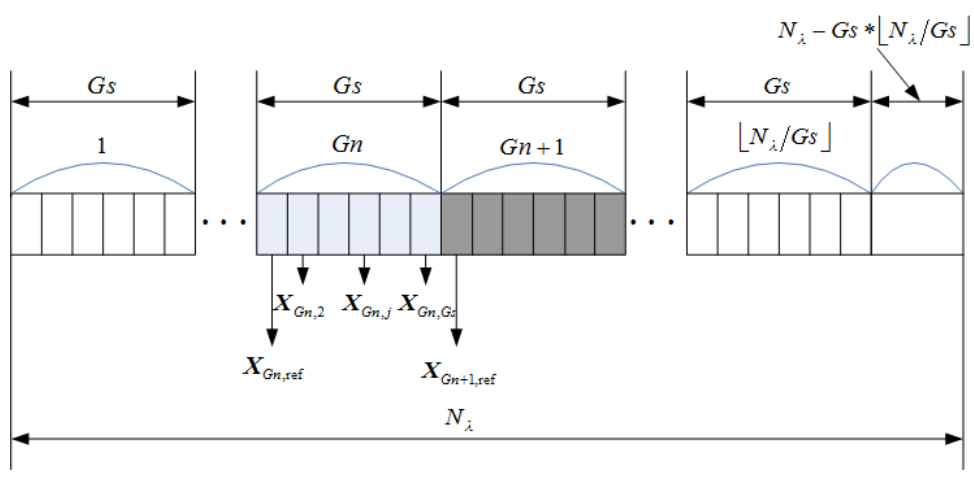

图 1 波段分组示意图

Fig.1 Band grouping diagram 


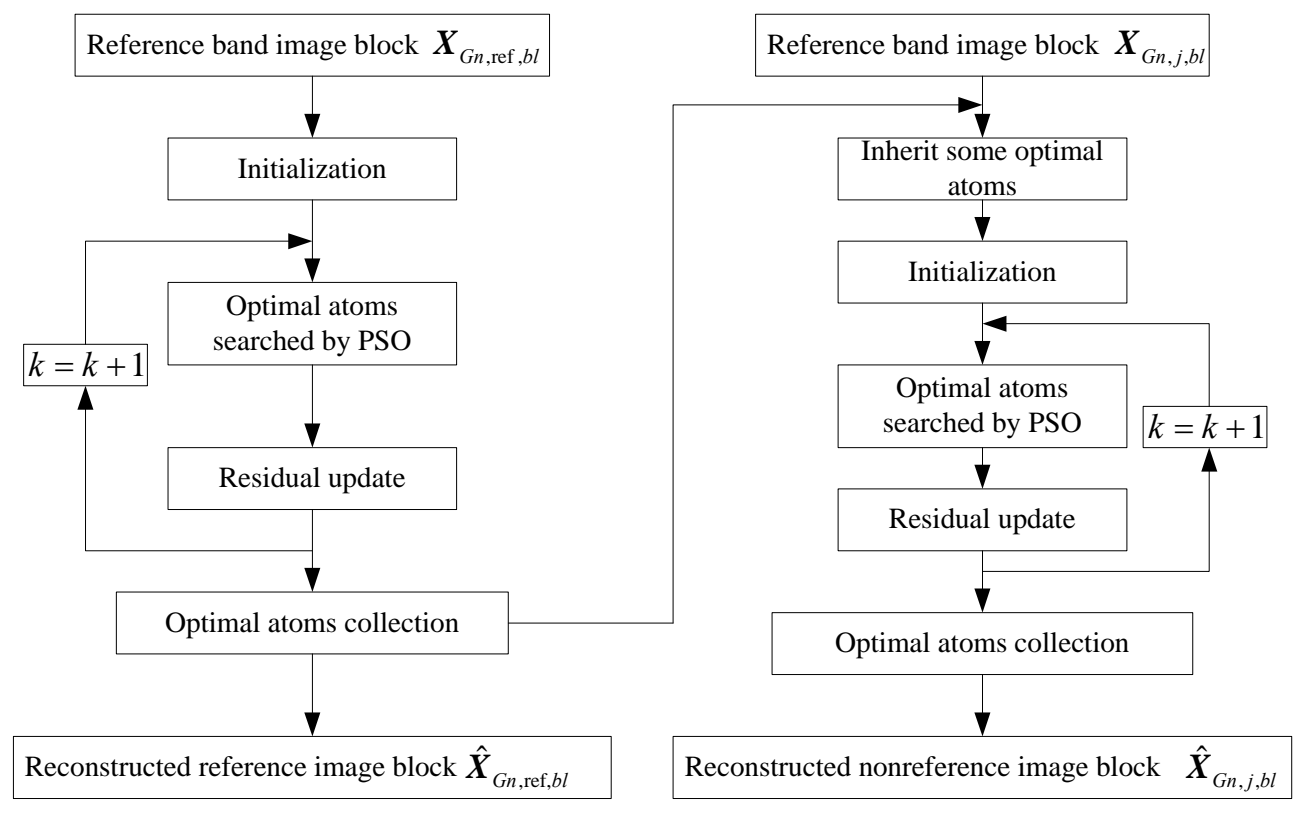

图 2 基于谱间相关性的稀疏分解算法框架

Fig.2 Framework of sparse decomposition algorithm based on spectral correlation

式中: round(.)表示取整; $\sigma$ 是继承系数; $h$ 是非参考波段图像与参考波段图像的谱间相关性, 计算公式为:

$$
h=\frac{\sum_{n_{\mathrm{r}}=1}^{B} \sum_{n_{\mathrm{c}}=1}^{B}\left[\boldsymbol{X}_{G \mathrm{n}, \text { ref }, b l}^{\prime}\left(n_{\mathrm{r}}, n_{\mathrm{c}}\right)-\overline{\boldsymbol{X}}_{G \mathrm{n}, \text { ref }, b l}^{\prime}\right]\left[X_{G \mathrm{n}, j, b l}^{\prime}\left(n_{\mathrm{r}}, n_{\mathrm{c}}\right)-\bar{X}_{G \mathrm{n}, j, b l}^{\prime}\right]}{\sqrt{\left(\sum_{n_{\mathrm{r}}=1}^{B} \sum_{n_{\mathrm{c}}=1}^{B}\left[\boldsymbol{X}_{G \mathrm{n}, \mathrm{ref}, b l}^{\prime}\left(n_{r}, n_{c}\right)-\bar{X}_{G \mathrm{n}, \mathrm{ref}, b l}^{\prime}\right]^{2}\right)\left(\sum_{n_{\mathrm{r}}=1}^{B} \sum_{n_{\mathrm{c}}=1}^{B}\left[\boldsymbol{X}_{G \mathrm{n}, j, b l}^{\prime}\left(n_{\mathrm{r}}, n_{\mathrm{c}}\right)-\bar{X}_{G \mathrm{n}, j, b l}^{\prime}\right]^{2}\right)}}
$$

式中: $\boldsymbol{X}_{G \mathrm{n}, \mathrm{ref}, b l}^{\prime}\left(n_{\mathrm{r}}, n_{\mathrm{c}}\right)$ 和 $\boldsymbol{X}_{G \mathrm{n}, j, b l}^{\prime}\left(n_{\mathrm{r}}, n_{\mathrm{c}}\right)$ 是 $X_{G \mathrm{n}, \mathrm{ref}, b l}$ 和 $X_{G \mathrm{n}, j, b l}$ 的矩阵形式, $\bar{X}_{G \mathrm{n}, \mathrm{ref}, b l}^{\prime}$ 和 $\bar{X}_{G \mathrm{n}, j, b l}^{\prime}$ 是图像块 $X_{G \mathrm{n}, \mathrm{ref}, b l}^{\prime}\left(n_{\mathrm{r}}, n_{\mathrm{c}}\right)$ 和 $X_{G \mathrm{n}, j, b l}^{\prime}\left(n_{\mathrm{r}}, n_{\mathrm{c}}\right)$ 像素值的均值, 利用 公式(3)和公式(4)进行计算:

$$
\bar{X}_{G \mathrm{n}, \mathrm{ref}, b l}^{\prime}=\frac{1}{B^{2}} \sum_{n_{\mathrm{r}}=1}^{B} \sum_{n_{\mathrm{c}}=1}^{B} \boldsymbol{X}_{G \mathrm{n}, \text { ref }, b l}^{\prime}\left(n_{\mathrm{r}}, n_{\mathrm{c}}\right)
$$$$
\left.\left[p_{0}, p_{1}, \ldots, p_{k}, \ldots, p_{K-1}\right]=\left[\left|\left\langle X_{G \mathrm{n}, j, b l}, g_{\gamma_{0}}\right\rangle\right|,\left|\left\langle X_{G \mathrm{n}, j, b l}, g_{\gamma_{1}}\right\rangle\right|, \cdots,\left|\left\langle X_{G \mathrm{n}, j, b l}, g_{\gamma_{k}}\right\rangle\right|, \cdots, \mid\left\langle X_{G \mathrm{n}, j, b l}, g_{\gamma_{K-1}}\right\rangle\right]\right]
$$

$$
\bar{X}_{G \mathrm{n}, j, b l}^{\prime}=\frac{1}{B^{2}} \sum_{n_{\mathrm{r}}=1}^{B} \sum_{n_{\mathrm{c}}=1}^{B} X_{G \mathrm{n}, j, b l}^{\prime}\left(n_{\mathrm{r}}, n_{\mathrm{c}}\right)
$$

确定继承个数 $K_{\text {in }}$ 后，计算非参考波段图像块 $X_{G \mathrm{n}, j, b l}$ 与参考波段图像块 $X_{G \mathrm{n}, \mathrm{ref}, \mathrm{bl}}$ 的最优原子 $\Theta_{K}=\left[g_{\gamma_{0}}, g_{\gamma_{2}}, \cdots, g_{\gamma_{k}}, \cdots, g_{\gamma_{K-1}}\right]$ 之间的相关性, 用公式

(5)表示:

的执行过程, 其他图像块与之相同, 总结如下:

\section{算法: MPSO_OMP}

输入: 第 $G n$ 组的参考波段图像块和非参考波段 段图像块从参考波段图像块继承得到的最优原子。计 算由这 $K_{\mathrm{in}}$ 个最优原子对非参考波段图像块 $X_{G \mathrm{n}, j, b l}$ 进 行稀疏表示后的残差，参见公式(6):

$$
R^{K_{\mathrm{in}}} X_{G \mathrm{n}, j, b l}=X_{G \mathrm{n}, j, b l}-\Theta_{K_{\mathrm{in}}}\left(\Theta_{K_{\mathrm{in}}}^{\mathrm{T}} \Theta_{K_{\mathrm{in}}}\right)^{-1} \Theta_{K_{\mathrm{in}}}^{\mathrm{T}} X_{G \mathrm{n}, j, b l}
$$

将此残差作为初始值, 利用 PSO 算法搜索 $K-$ $K_{\text {in }}$ 个原子, 得到表示非参考波段图像块的 $K$ 个最优 原子。

\section{3 算法主要步骤}

以单个图像块 $X_{G \mathrm{n}, j, b l}$ 为例说明算法 MPSO_OMP

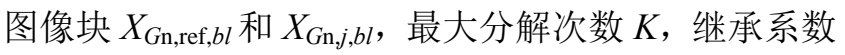
$\sigma$, 粒子群优化中的最大进化代数 $T_{\max }$ 和种群数量 $P_{\mathrm{op}}$, 参考波段图像块 $X_{G \mathrm{n}, \mathrm{ref}, b l}$ 的最优原子集合:

$$
\Theta_{K}=\left[g_{\gamma_{0}}, g_{\gamma_{2}}, \cdots, g_{\gamma_{k}}, \cdots, g_{\gamma_{K-1}}\right]
$$

输出: 非参考波段图像块的重构值 $\hat{X}_{G \mathrm{n}, j, b l}$

Step 1: 根据公式(1)计算 $X_{G \mathrm{n}, j, b l}$ 与 $X_{G \mathrm{n}, \mathrm{ref}, b l}$ 的谱间 相关性 $h$, 并计算继承个数 $K_{\text {in }}$;

Step 2 : 根据公式 (5) 计算 $X_{G \mathrm{n}, j, b l}$ 与 $\Theta_{K}=\left[g_{\gamma_{0}}, g_{\gamma_{2}}, \cdots, g_{\gamma_{k}}, \cdots, g_{\gamma_{K-1}}\right]$ 的相关性并进行排序, 确定 
继承得到的 $K_{\text {in }}$ 个最优原子:

$$
\Theta_{K_{\mathrm{in}}}=\left[g_{\gamma_{0}}^{\prime}, g_{\gamma_{2}}^{\prime}, \cdots, g_{\gamma_{k}}, \cdots, g_{\gamma_{K_{\mathrm{in}}-1}}\right] ;
$$

Step 3: 根据公式(6)确定初始残差 $R^{K_{\mathrm{in}}} X_{G \mathrm{n}, j, b l}$, 最 优原子集合 $\Theta_{K_{\mathrm{in}}}=\left[g_{\gamma_{0}}^{\prime}, g_{\gamma_{2}}^{\prime}, \cdots, g_{\gamma_{k}}, \cdots, g_{\gamma_{\mathrm{K}_{\mathrm{in}}-1}}\right]$, 分解次数 $k=1$;

Step 4: 利用 PSO 寻找最优原子, 达到最大进化 代数 $T_{\max }$ 后立即终止搜索, 输出 $G_{\mathrm{best}}$;

Step 5 : 利用 $G_{\text {best }}$ 更新最优原子集合, $\Theta_{K_{\text {in }}+k}=\Theta_{K_{\text {in }}+k-1} \mathrm{U} g_{G_{\text {best }}}$, 其中, $g_{G_{\text {best }}}$ 是由 $G_{\text {best }}$ 产生的 原子;

Step 6: 更新残差

$$
R^{K_{\mathrm{in}}+k} X_{G \mathrm{n}, j, b l}=X_{G \mathrm{n}, j, b l}-\Theta_{K_{\mathrm{in}}+k}\left(\Theta_{K_{\mathrm{in}}+k}^{\mathrm{T}} \Theta_{K_{\mathrm{in}}+k}\right)^{-1} \Theta_{K_{\mathrm{in}}+k}^{\mathrm{T}} X_{G \mathrm{n}, j, b l} ;
$$

Step 7: 判断是否达到最大分解次数 $K-K_{\text {in }}$, 若 不满足则 $k=k+1$, 重复 Step 4 Step 6; 若满足则停 止迭代。

Step 8: 计算由最优原子集合 $\Theta_{K}$ 对 $X_{G \mathrm{n}, j, b l}$ 进行稀 疏表示得到的重构 $\hat{X}_{G \mathrm{n}, j, b l}$ :

$$
\hat{X}_{G \mathrm{n}, j, b l}=X_{G \mathrm{n}, j, b l}-\Theta_{K}\left(\Theta_{K}^{\mathrm{T}} \Theta_{K}\right) \Theta_{K}^{\mathrm{T}} X_{G \mathrm{n}, j, b l}
$$

\section{2 实验结果与分析}

\section{1 高光谱数据集}

选择 4 组高光谱图像评估稀疏分解算法的性能, 分别是来自 AVIRIS 采集的 Cuprite 1、Cuprite 2、Indian Pines 以及 ROSIS 采集的 Pavia University。原始数据 集中的水汽波段和噪声波段均已移除, 且根据分块大 小 $B=16$ 对图像进行了空间剪裁, 4 组数据的基本情 况见表 1 。高光谱数据第 50 个波段的原始图像如图 3 所示。

\section{表 1 四组高光谱数据集的基本情况}

Table 1 Four hyperspectral datasets

\begin{tabular}{lllll}
\hline \multirow{2}{*}{ Dataset } & $\begin{array}{l}\text { Original } \\
\text { bands }\end{array}$ & $\begin{array}{l}\text { Original } \\
\text { image size }\end{array}$ & $\begin{array}{l}\text { Available } \\
\text { bands }\end{array}$ & $\begin{array}{l}\text { Cropped } \\
\text { image size }\end{array}$ \\
\hline Cuprite1 & 224 & $614 \times 512$ & 188 & $256 \times 256$ \\
Cuprite2 & 224 & $614 \times 512$ & 188 & $256 \times 256$ \\
Indian Pines & 220 & $145 \times 145$ & 200 & $128 \times 128$ \\
Pavia & 115 & $610 \times 340$ & 103 & $256 \times 256$ \\
University & & & & \\
\hline
\end{tabular}

\section{2 实验参数设置}

不论是利用 OMP 算法、PSO_OMP 算法还是算 法 MPSO_OMP 对高光谱图像进行稀疏分解, 均需设 定最大分解次数, 该参数对算法的时间复杂度及重构 图像的精度具有重要影响。所提算法 MPSO_OMP 还
需设定的参数是粒子群优化中的最大进化代数和种 群大小, 以及继承系数。分组大小 $G_{\mathrm{s}}$ 的选择会影响 算法性能，通常来说， $G_{\mathrm{s}}$ 越小，组内的谱间相关性越 强，非参考波段图像块能够从参考波段图像块继承到 更多的最优原子, 稀疏分解效率越高, 但此时分组个 数也较多，时间复杂度也会增加。当高光谱成像仪的 光谱分辨率较高时, 相对较大的分组也能保证组内图 像的强相关性, 重构精度也能得到保证。根据实验场 景对应的传感器的光谱分辨率, 同时考虑到计算复杂 度, 分组大小选择为 $G_{\mathrm{s}}=8$ 。

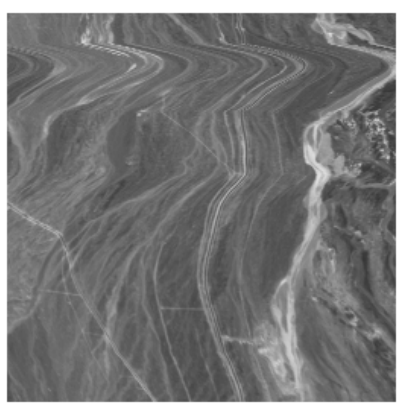

(a) Cuprite 1

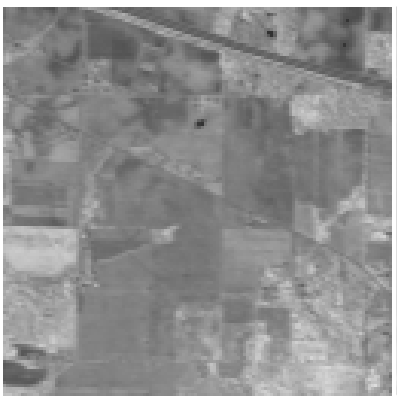

(c) Indian Pines

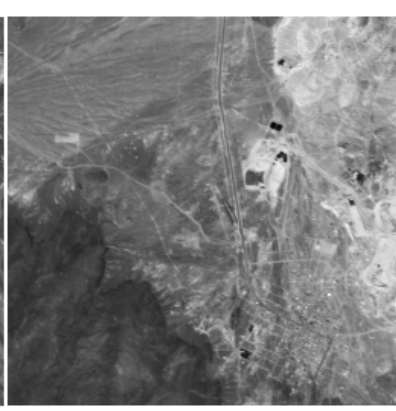

(b) Cuprite 2

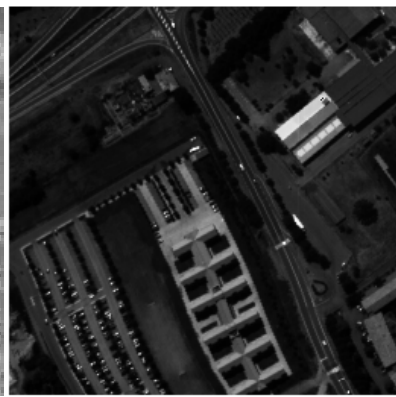

(d) Pavia University
图 3 四组高光谱数据的第 50 个波段的原始图像

Fig.3 Original 50th band images of four hyperspectral datasets

利用重构图像的峰值信噪比 (peak signal-to- noise ratio, PSNR) 和运行时间对算法性能进行评价。实验 的运行软硬件环境为: AMD 四核 CPU, $3.80 \mathrm{GHz}$, $16 \mathrm{G}$ 内存, Matlab2012b。单个图像块的重构 PSNR 计算公式为:

$$
\operatorname{PSNR}\left(X_{G \mathrm{n}, j, b l}, \hat{X}_{G \mathrm{n}, j, b l}^{k}\right)=20 \times \lg \frac{\max \left(X_{G n, j, b l}\right)}{\sqrt{\operatorname{MSE}\left(X_{G \mathrm{n}, j, b l}, \hat{X}_{G \mathrm{n}, j, b l}^{k}\right)}}
$$

式中: $\hat{X}_{G \mathrm{n}, j, b l}^{k}$ 是利用 $k$ 个最优原子得到的重构图像块 的向量化表示; $\max \left(X_{G \mathrm{n}, j, b l)}\right)$ 是原始图像块 $X_{G \mathrm{n}, j, b l}$ 的峰 值; $\operatorname{MSE}\left(X_{G \mathrm{n}, j, b l}, \hat{X}_{G \mathrm{n}, j, b l}^{k}\right)$ 是均方误差, 定义为:

$$
\operatorname{MSE}\left(X_{G \mathrm{n}, j, b l}, \hat{X}_{G \mathrm{n}, j, b l}^{k}\right)=\frac{1}{N}\left\|X_{G \mathrm{n}, j, b l}-\hat{X}_{G \mathrm{n}, j, b l}^{k}\right\|_{2}^{2}
$$




\subsection{1 粒子群参数的确定}

首先利用算法 PSO_OMP 对 4 组高光谱数据的第 50 个波段图像进行稀疏分解, 分析最大进化代数、种 群大小和分解次数对算法性能的影响。粒子群优化中 最大进化代数 $T_{\max }$ 的变化范围是 $5 \sim 50$, 间隔是 5 , 种群大小 $P_{\mathrm{op}}$ 的变化范围是 $5 \sim 50$, 间隔是 5 , 分解次 数 $K$ 的变化范围是 $10 \sim 100$, 间隔是 10 。同一参数下 算法 PSO_OMP 运行 10 次以消除算法的随机性, 图 4 给出的是 Cuprite 2 重构图像的平均 PSNR 随参数的变 化。分解次数为 50 次时, 平均重构 PSNR 随最大进 化代数和种群大小的变化如图 4(a)所示。同一种群个 数下, PSNR 随着最大进化代数的增加没有大幅度的 增加, 即最大进化代数对 PSNR 的影响不大。同一最 大进化代数下, 随着种群个数的增加, PSNR 以较慢 的速度逐渐增加。

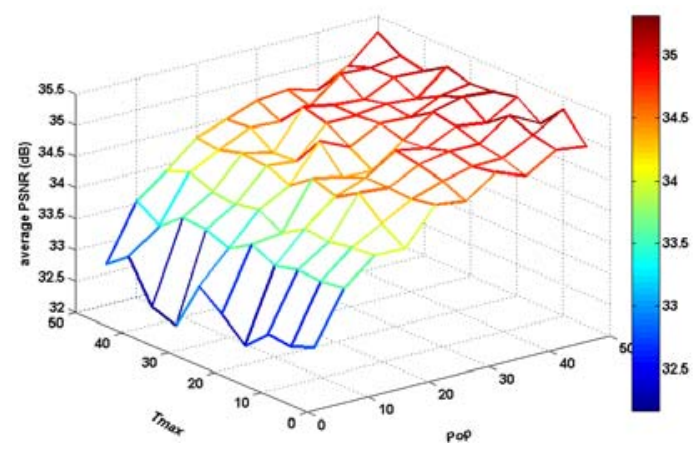

（a) 分解次数为 50 , 最大进化代数和种群大小对重构 PSNR 的 影响

(a) Influence of maximum evolution agebra and population size on reconstructed PSNR, when the decomposition number is 50

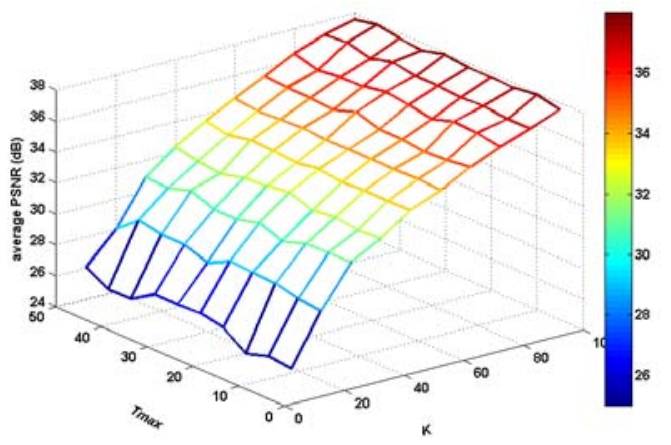

（b) 种群大小为 10 , 最大进化代数和分解次数对重构 PSNR 的 影响

(b) Influence of maximum evolution agebra and decomposition number on reconstructed PSNR, when the population size is 10

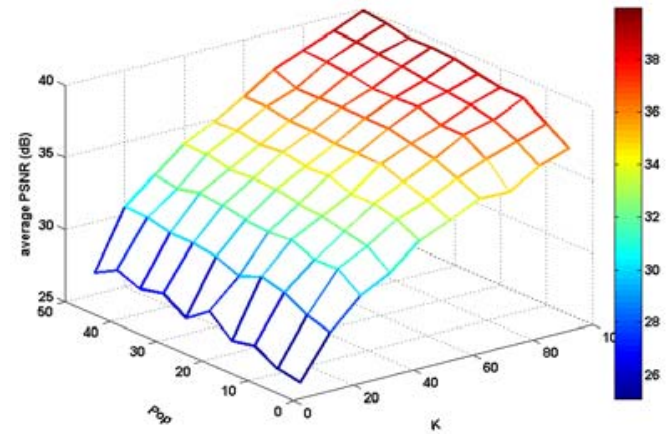

（c) 最大进化代数为 5 , 种群大小和分解次数对重构 PSNR 的 影响

(c) Influence of population size and decomposition number on reconstructed PSNR, when the maximum evolution agebra is 5

图 4 最大进化代数、种群大小和分解次数对 PSO_OMP 算法 的影响

Fig.4 Influence of maximum evolution agebra, population size and decomposition number on PSO_OMP

种群大小为 10 时, 最大进化代数和分解次数对 重构 PSNR 的影响如图 4(b)所示。同一分解次数下, PSNR 随着最大进化代数的增加仅有小幅度的振荡, 进一步地表明最大进化代数对重构精度的影响不大。 同一最大进化代数下, 随着分解次数的增加, PSNR 不断增加, 且与图 4(a)中种群个数增加所引起的 PSNR 增长相比, 分解次数的增加更能令 PSNR 快速增长。 最大进化代数为 5 时, 种群大小和分解次数对重构性 能的影响如图 4(c)所示。同一分解次数下, PSNR 随着 种群数量的增加会不断增加, 但增长的速度较慢。同 一种群数量下, 随着分解次数的增加, PSNR 会逐渐增 加, 增长速度快于种群数量增加所引起的 PSNR 增长, 进一步地说明增加分解次数更有助于提高 PSNR。

当最大进化代数和种群个数逐渐增大时, 粒子群 优化过程将需要从更多的粒子中经过多次进化才能 找到最优原子, 即在二者的影响下, 时间复杂度均会 不断增加。其他 3 组高光谱图像的实验结果与 Cuprite2 相似, 综合考虑重构精度和计算复杂度, 将粒子群优 化的最大进化代数设置为 $T_{\max }=5$, 种群大小设置为 $P_{\mathrm{op}}=10$ 。

\subsection{2 分解次数的确定}

利用 PSO_OMP 算法和 OMP 算法对 4 组高光谱 数据集的第 50 个波段图像进行稀疏分解, 设定算法 在分解次数达到 150 次时终止。图 5 给出两种算法得 到的重构 PSNR 随分解次数的变化, 其中粗线是 OMP 分解次数达到 50 次时, 利用 50 个最优原子对原始图 像进行稀疏表示后, 得到的重构图像的精度。实验结 果表明, 算法PSO_OMP仅用 50 个原子无法达到 OMP 算法的重构精度, 这是因为: OMP 算法的每次分解 
过程中, 能够从咒余字典的所有原子中寻找到与残差 最匹配的原子, 性能得到保证。粒子群算法具有随机 性, 每次分解寻找到的最优原子可能并不是与残差最 匹配的原子, 因此需要寻找到更多的原子才能充分表 征原始图像的特征, 才能达到 OMP 算法的重构精度。 如果将 OMP 算法利用 50 个原子得到的重构图像的 PSNR 作为标准, Cuprite1 和 Cuprite2 大约需要 80 个 原子左右才能达到此标准, 而 Indian Pines 和 Pavia University 大约需要 120 个甚至更多的原子才能达到 与 $\mathrm{OMP}$ 算法相同的精度。据此, OMP 算法的最大分 解次数设定为 $K=50$, 针对 4 组高光谱数据集, 算法 PSO_OMP 的最大分解次数分别设定为 $K=[100,100$, $150,150]$ 。

\subsection{3 继承系数的确定}

针对非参考波段图像块 $X_{G \mathrm{n}, j, b l}$, 将残差 $R^{K_{\mathrm{in}}} X_{G \mathrm{n}, j, b l}$

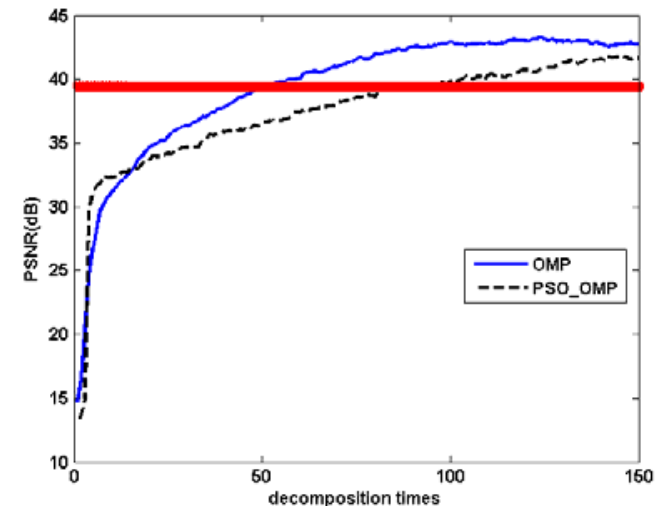

(a) Cuprite 1 的重构 PSNR 随分解次数的变化

(a) Reconstructed PSNR vs decomposition number for Cuprite 1

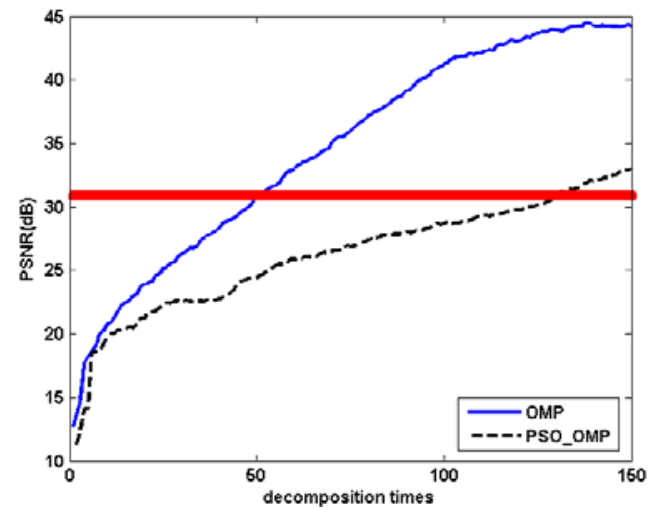

(c) Indian Pines 的重构 PSNR 随分解次数的变化

(c) Reconstructed PSNR vs decomposition number for Indian Pines
作为初始值进行稀疏分解, 则最大进化代数、种群个 数及最大分解次数对 MPSO_OMP 算法性能的影响应 该与其对 PSO_OMP 算法性能的影响一致, 故将 MPSO_OMP 的参数设定为: $T_{\max }=5, P_{\mathrm{op}}=10$ 和 $K$ $=[100,100,150,150]$ 。

对非参考波段图像进行稀疏分解时, 继承系数的 选择是影响 MPSO_OMP 算法性能的主要因素。利用 MPSO_OMP 算法对 4 组高光谱数据集的第 5 个分组 内（即波段序号为 41 48）的所有波段图像进行稀疏 分解, 分析继承系数对重构性能的影响。继承系数的 变化范围是 $0 \sim 1$, 间隔为 0.1 。图 6 给出不同继承系 数下, Cuprite1 和 Indian Pines 两个高光谱数据集的平 均重构 PSNR 及运行时间。

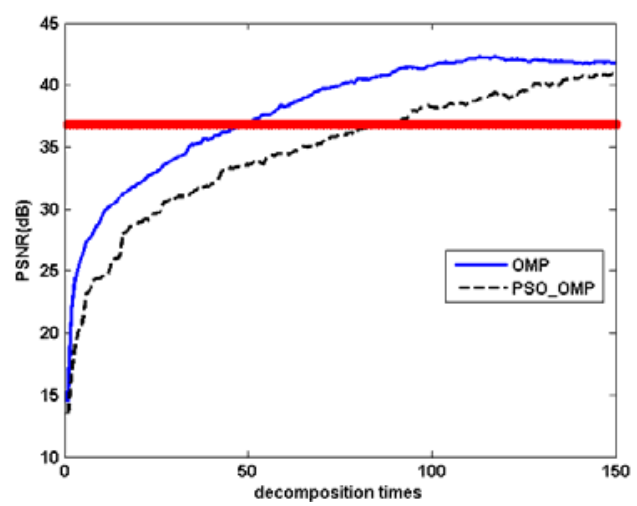

(b) Cuprite 2 的重构 PSNR 随分解次数的变化

(b) Reconstructed PSNR vs decomposition number for Cuprite 2

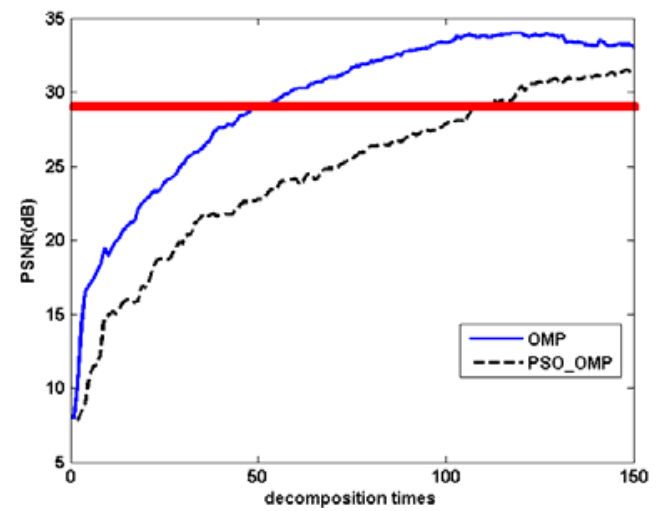

(d) Pavia University 的重构 PSNR 随分解次数的变化

(d) Reconstructed PSNR vs decomposition number for Pavia University

图 5 OMP 算法和 PSO_OMP 算法的重构 PSNR 随分解次数的变化

Fig.5 Reconstructed PSNR vs decomposition number of algorithm OMP and algorithm PSO_OMP 
当谱间相关性 $h$ 确定时, 继承系数越小, 继承得 到的最优原子个数越少, 粒子群所需搜索的原子个数 越多, 此时可用更多的原子去表示图像的差异性, 重 构性能较好。当继承系数逐渐增大时, 从参考波段图 像的最优原子集合中继承的原子个数越多, 粒子群所 需搜索的原子个数越少, 重构性能会出现一定程度的 下降。在继承系数逐渐增大的过程中, 因粒子群算法 具有随机性, 重构 PSNR 会出现不同程度的振荡。整 体来说, 重构 PSNR 的下降速度较慢, 从继承系数为 0 增加至继承系数为 1 时, 重构 PSNR 的下降值保持 在 $1 \mathrm{~dB}$ 左右, 说明算法仍能保证重构精度。

从计算复杂度来看, MPSO_OMP 算法的计算复 杂度主要由继承原子的选择过程与其他原子的粒子 群搜索过程决定。与继承原子的选择过程相比, 粒子 群搜索过程更耗时。因此, 随着继承系数的增大, 继 承原子数不断增多, 粒子群搜索的原子数不断降低, 则算法的计算复杂度会逐渐降低, 如图 6 所示。综合 考虑重构性能和重构计算复杂度, 当继承系数选择为 0.5 时, 重构 PSNR 的下降幅度保证在 $0.4 \mathrm{~dB}$ 以内, 而运行速度却能提高 1.3 倍左右, 故将 MPSO_OMP

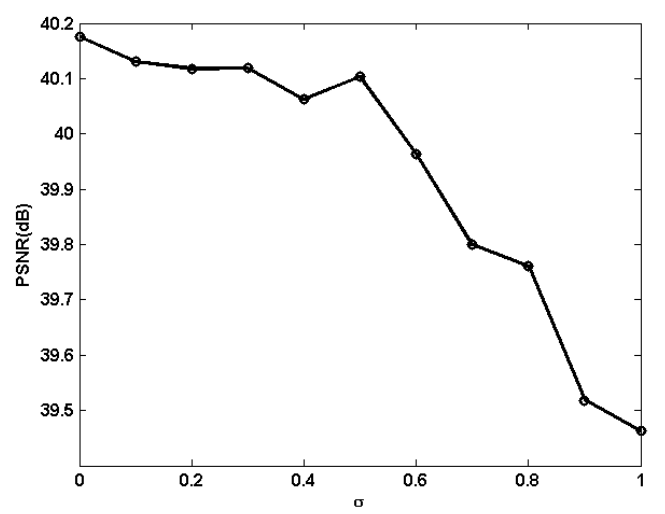

(a) Cuprite 1 的重构 PSNR (a) Reconstructed PSNR for Cuprite 1

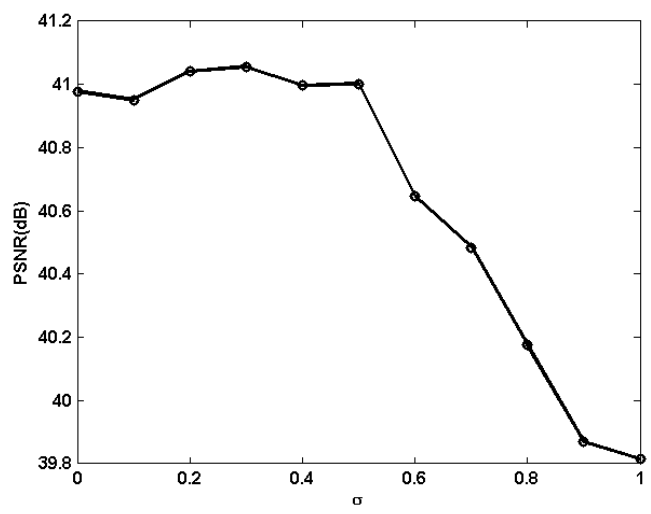

(c) Indian Pines 的重构 PSNR

(c) Reconstructed PSNR for Indian Pines
算法中的继承系数确定为 $\sigma=0.5$ 。

\section{3 实验结果分析}

利用 OMP 算法、PSO_OMP 算法和 MPSO_OMP 算法对 4 组高光谱图像进行稀疏分解, 分析算法的性 能。OMP 算法是指对所有波段图像采用 OMP 进行稀 疏分解, 最大分解次数 $K=50$ 。PSO_OMP 算法是指 对所有波段图像采用 PSO_OMP 算法进行稀疏分解, 最大分解次数 $K=[100,100,150,150]$, 最大进化代数 $T_{\text {max }}=5$, 种群大小 $P_{\mathrm{op}}=10$ 。MPSO_OMP 算法是指对 高光谱图像进行分组后, 参考波段图像采用 PSO_OMP 算法进行稀疏分解, 非参考波段图像采用 MPSO_OMP 算法进行稀疏分解, 最大分解次数 $K=$ $[100,100,150,150]$, 继承系数 $\sigma=0.5$, 粒子群中的最 大进化代数 $T_{\mathrm{max}}=5$, 种群大小 $P_{\mathrm{op}}=10$ 。表 2 给出 3 种算法的实验结果, 表中的平均 PSNR 是高光谱数据 集所有波段图像重构 PSNR 的均值, 运行时间是完成 单个波段稀疏分解所需的平均时间, 加速比是指以 OMP 算法的稀疏分解速度为标准, 算法 PSO_OMP 和算法 MPSO_OMP 的加速倍数。

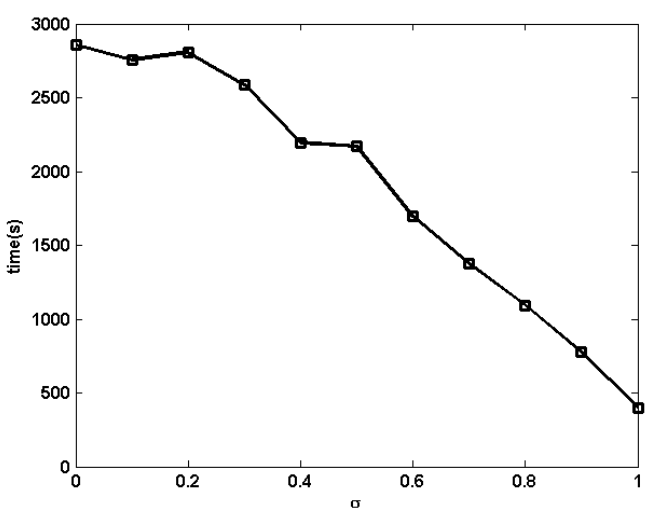

(b) Cuprite 1 的运行时间 (b) Runtime for reconstructing Cuprite 1

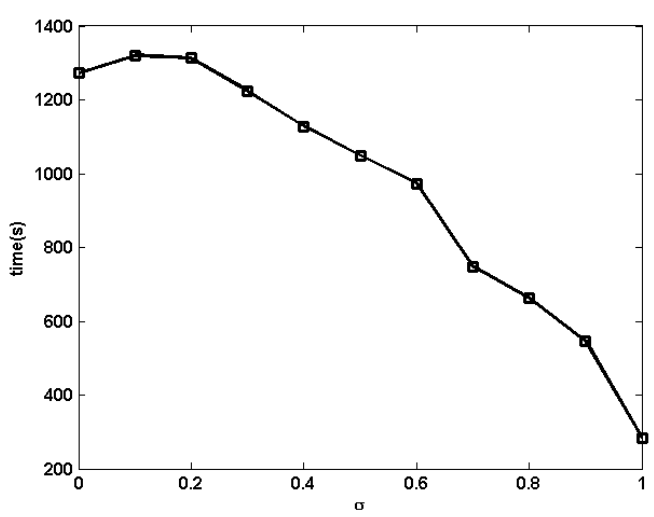

(d) Indian Pines 的运行时间

(d) Runtime for reconstructing Indian Pines

图 6 继承系数对算法 MPSO_OMP 的影响

Fig.6 Influence of inheritance coefficient on MPSO_OMP 
表 2 OMP、PSO_OMP 和 MPSO_OMP 的性能对比

Table 2 Performance comparison between OMP, PSO_OMP

\begin{tabular}{llll}
\multicolumn{4}{c}{ and proposed MPSO_OMP } \\
\hline Algorithm & $\begin{array}{l}\text { Average } \\
\text { PSNR/dB }\end{array}$ & Run time/s & $\begin{array}{l}\text { Acceleration } \\
\text { ratio }\end{array}$ \\
\hline Cuprite1 & & & \\
\hline OMP & 40.21 & 5771 & 1 \\
PSO_OMP & 40.30 & 290 & 20 \\
MPSO_OMP & 40.13 & 177 & 33 \\
\hline Cuprite2 & & & \\
\hline OMP & 39.69 & 5372 & 1 \\
PSO_OMP & 39.66 & 293 & 18 \\
MPSO_OMP & 39.46 & 188 & 29 \\
\hline Indian Pines & & & 1 \\
\hline OMP & 31.89 & 1478 & 13 \\
PSO_OMP & 33.78 & 110 & 19 \\
MPSO_OMP & 33.63 & 79 & 13 \\
\hline Pavia University & & & 21 \\
\hline OMP & 39.07 & 5646 & \\
PSO_OMP & 42.69 & 440 & 268 \\
MPSO_OMP & 42.59 & & 13 \\
\hline
\end{tabular}

算法 PSO_OMP 的重构 PSNR 与 OMP 算法的 PSNR 相当或略高于后者, 这充分说明, 利用粒子群 的进化过程可以模拟 OMP 算法的匹配过程, 搜索得 到最优原子并实现图像的稀疏分解。因粒子群算法具 有随机性, 每次分解得到的原子可能并不是最优的, 在保证相同重构精度的条件下, 最优原子个数要多于 OMP 算法所需搜索的原子个数。但从计算复杂度分 析, PSO_OMP 算法具有较大的优势, 与 OMP 算法 相比, 运行时间能降低一个数量级, 计算效率提高 10 倍以上。

对场景 Cuprite 1 和 Cuprite 2, 利用算法 MPSO_OMP 得到的重构 PSNR 与 OMP 算法的 PSNR 相当。这充分说明, 在对非参考波段图像进行稀疏分 解时, 从参考波段图像的最优原子集合中继承部分原 子的方法能够表示图像的共性特征，利用谱间相关性 对 PSO_OMP 进行改进具有可靠性。与粒子群搜索过 程的计算复杂度相比, 继承原子的选择过程耗时较 少, 因此这种改进方法能更进一步地降低整个稀疏分 解过程的计算复杂度。对于场景 Indian Pines 来说, 利用 PSO_OMP 算法和 MPSO_OMP 算法均能保证重 构精度, 与 PSO_OMP 算法相比, MPSO_OMP 算法 能够进一步地提高稀疏分解效率。但因 Indian Pines 数据的谱间相关性要弱于其他 3 组高光谱数据, 因此
MPSO_OMP 的加速最不明显。对于场景 Pavia University 来说, 利用 150 个原子得到的重构图像的 PSNR 要高出利用 50 个原子得到的重构 PSNR, 且运 算效率有显著提高。

数据集 Cuprite1 经过稀疏分解后, 重构图像与原 始图像的对比如图 7 所示, 图中为原始图像和重构图 像的第 40 个波段（该波段是非参考波段），并给出 了相应的重构 PSNR。对于场景 Cuprite1 来说, 3 种 算法得到的重构 PSNR 相差无几, OMP 算法、 PSO_OMP 算法和 MPSO_OMP 算法的重构 PSNR 分 别能达到 $40.1143 \mathrm{~dB} 、 40.1518 \mathrm{~dB}$ 和 $40.1194 \mathrm{~dB}$ 。重 构图像能够很好地描述原始图像的细节特征, 充分说 明利用 PSO 算法去优化 OMP 算法的匹配过程非常可 靠。PSO_OMP 算法和 MPSO_OMP 算法得到的重构 图像非常接近, 说明继承原子能够表示图像的共性特 征, 而粒子群搜索的其他部分原子表示了图像的差异 性, 二者相互结合, 共同完成图像的高精度稀疏分解, 充分说明算法的有效性。

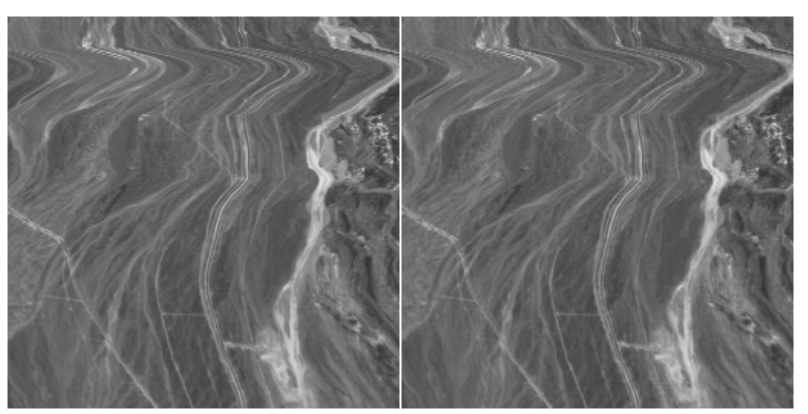

(a) 原始图像

(b) OMP 重构图像

$\begin{array}{ll}\text { (a) Original image } & \text { (b) Reconstructed image by OMP }\end{array}$

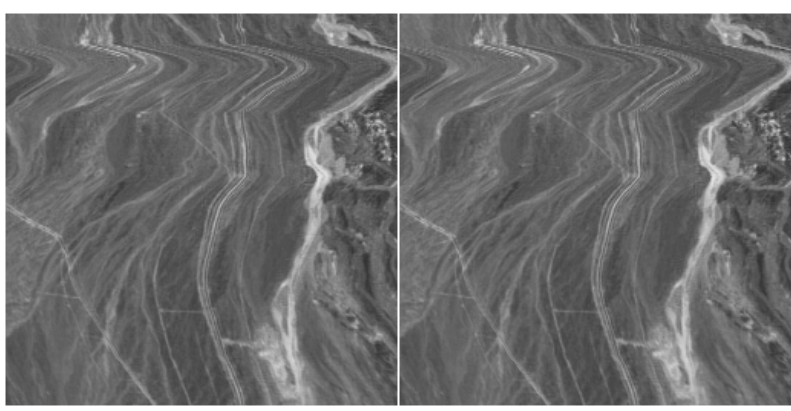

(c) PSO_OMP 重构图像

(d)MPSO_OMP 重构图像

(c) Reconstructed image by PSO_OMP

(d) Reconstructed image by MPSO_OMP

图 7 数据集 Cuprite1 的重构图像与原始图像对比

Fig.7 Comparison between original image and reconstructed images of Cuprite1

\section{3 结论}

提出了基于谱间相关性的稀疏分解算法，在利用 
粒子群优化对 OMP 算法匹配过程加速的基础上; 考 虑参考波段图像和非参考波段图像的谱间相关性，采 用继承部分最优原子的方式改进非参考波段图像的 最优原子搜索算法, 进一步地提高稀疏分解效率。实 验结果表明, 与 OMP 算法及 PSO_OMP 算法相比, 在保证重构精度的前提下, 所提算法 MPSO_OMP 能 够进一步有效地提高稀疏分解过程的计算效率。因粒 子群算法存在一定的随机性, 如何降低随机性对算法 性能的影响将是下一步的工作方向。

\section{参考文献:}

[1] NIU Y, WANG B. Extracting Target Spectrum for hyperspectral target detection: an adaptive weighted learning method using a self-completed background dictionary[J]. IEEE Transactions on Geoscience and Remote Sensing, 2017, 55(3): 1604-1617. (DOI: 10.1109/TGRS.2016.2628085)

[2] 蔡庆空, 李二俊, 蒋金豹, 等. 联合光谱-空间信息的短波红外高光谱 图像茶叶识别模型 [J]. 光谱学与光谱分析, 2019, 39(8): 2522-2527.

CAI Q, LI E, JIANG J, et al. Study on the tea identification of near-infrared hyperspectral image combining spectra-spatial information[J]. Spectroscopy and Spectral Analysis, 2019, 39(8): 2522-2527.

[3] 陈善学, 周艳发, 漆若兰. 基于核函数的联合稀疏表示高光谱图像分 类[J]. 系统工程与电子技术，2018，40(3)：692-698. (DOI: 10.3969 /j.issn. 1001-506X).

CHEN S, ZHOU Y, QI R. Joint sparse representation of hyperspectral image classification based on kernel function[J]. Systems Engineering and Electronics, 2018, 40(3): 692-698. (DOI: 10.3969 /j.issn. 1001506X).

[4] FANG L, HE N, LI S, et al. A new spatial-spectral feature extraction method for hyperspectral images using local covariance matrix representation[J]. IEEE Transactions on Geoscience and Remote Sensing, 2018, 56(6): 3534-3546. (DOI: 10.1109/ TGRS.2018.2801387).

[5] 张淳民, 穆廷魁, 颜廷昱, 等. 高光谱遥感技术发展与展望[J]. 航天返 回与遥感, 2018, 39(3): 104-114. (DOI:10.3969/j.issn.1009-8518).

ZHANG C, MU T, YAN T, et al. Overview of hyperspectral remote sensing technology[J]. Spacecraft Recovery \& Remote Sensing, 2018, 39(3): 104-114. (DOI:10.3969/j.issn.1009-8518).

[6] CHANG C I. A review of virtual dimensionality for hyperspectral imagery[J]. IEEE Journal of Selected Topics in Applied Earth Observations and Remote Sensing, 2018, 11(4): 1285-1305. (DOI: 10.1109/JSTARS. 2017.2782706).

[7] 唐中奇, 付光远, 陈进, 等. 基于低秩结构提取的高光谱图像压缩表 示[J]. 电子与信息学报, 2016, 38(5): 1085-1091.

TANG Z, FU G, CHEN J, et al. Low-rank structure based hyperspectral compression representation[J]. Journal of Electronics \& Information Technology, 2016, 38(5): 1085-1091.

[8] 马馨宏, 郭树旭. 高光谱遥感图像的稀疏分解与压缩感知[J]. 吉林大 学学报: 理学版, 2015, 53(4): 767-772.

MA X, GUO S. Compressed sensing and sparse decomposition of hyperspectral imgae[J]. Journal of Jilin University: Science Edition, 2015, 53(4): 767-772.

[9] GUO K, XIE X, XU X, et al. Compressing by learning in a low-rank and sparse decomposition form[J]. IEEE Access, 2019, 7: 150823-150832.

[10] 王忠良, 冯文田, 粘永健. 结合光谱解混与压缩感知的高光谱图像有 损压缩[J]. 红外与激光工程, 2018, 47(S1): 197-204.

WANG Z, FENG W, NIAN Y. Compressive- sensing-based lossy compression for hyperspectral images using spectral unmixing[J]. Infrared and Laser Engineering, 2018, 47(S1): 197-204.

[11] YAO L, DU X. Identification of underwater targets based on sparse representation[J]. IEEE Access, 2020(8): 215-228.

[12] TAN Shoubiao, SUN Xi, CHAN Wentao, et al. Robust face recognition with kernelized locality-sensitive group sparsity representation[J]. IEEE Transactions on Image Processing, 2017, 26(10): 4661-4668.

[13] PAN L, LI H C, MENG H, et al. Hyperspectral image classification via low-rank and sparse representation with spectral consistency constraint [J]. IEEE Geoscience and Remote Sensing Letters, 2017, 14(11): 2117-2121.

[14] 任智伟, 吴玲达. 基于本征图像分解的高光谱图像空谱联合分类[J]. 航天返回与遥感, 2019, 40(3): 111-120. (DOI:10.3969/j.issn.10098518).

REN Z, WU L. Spectral-spatial classification for hyperspectral imagery based on intrinsic image decomposition[J]. Spacecraft Recovery \& Remote Sensing, 2019, 40(3): 111-120. (DOI:10.3969/j.issn.1009-8518).

[15] SONG X, WU L and HAO H. Hyperspectral image denoising base on adaptive sparse representation[C]//2018 IEEE Third International Conference on Data Science in Cyberspace (DSC), 2018: 735-739.

[16] MEI S, HOU J, CHEN J, et al. Simultaneous spatial and spectral low-rank representation of hyperspectral images for classification[J]. IEEE Transactions on Geoscience and Remote Sensing, 2018, 56(5): 2872-2886.

[17] TROPP J A, GILBERT A C. Signal recovery from random measurements via orthogonal matching pursuit[J]. IEEE Transactions on Information Theory, 2007, 53(12): 4655-4666.

[18] Kulkarni A, Mohsenin T. Low overhead architectures for OMP compressive sensing reconstruction algorithm[C]//IEEE Transactions on Circuits and Systems I: Regular Papers, 2017, 64(6): 1468-1480.

[19] CHEN S, DONOHO D, Saunders M. Atomic decomposition by basis pursuit[J]. SIAM Journal on Scientific Computing, 1999, 20: 33- 61.

[20] 王丽, 冯燕. 基于粒子群优化的图像稀疏分解算法研究 [J]. 计算机仿 真, 2015, 32(11): 363-367.

WANG L, FENG Y. Sparse decomposition of images based on particle swarm optimization[J]. Computer Simulation, 2015, 32(11): 363-367.

[21] 王丽, 冯燕. 基于空谱联合的多假设预测高光谱图像压缩感知重构 算法[J]. 电子与信息学报, 2015, 37(12): 3000-3008.

WANG L, FENG Y. Compressed sensing reconstruction of hyperspectral images based on spatial-spectral multihypothesis prediction[J]. Journal of Electronics \& Information Technology, 2015, 37(12): 3000-3008. 